\title{
Trombocitopenia inmune. Guía de diagnóstico y tratamiento
}

\author{
Guideline for diagnosis and treatment of immune \\ thrombocytopenia
}

\author{
Comité Nacional de Hematología, Sociedad Argentina de Pediatría. \\ Dr. Hugo Donato, Dra. Viviana Bacciedoni, Dra. María Cristina Rapetti, \\ Dra. Graciela Elena, Dra. Marta Lavergne, Dr. Néstor Rossi, Dra. Alejandra Cedola, \\ Dr. Rodrigo Parias Nucci
}

\begin{abstract}
RESUMEN
El manejo de la trombocitopenia inmune es motivo de discusión en lo concerniente a evolución, diagnóstico, pronóstico y tratamiento. Se han publicado varias guías que expresan distintas opiniones de expertos, pero no existe aún consenso mundial sobre cuál es el manejo más adecuado de la enfermedad. Esta guía establece los criterios para definir el diagnóstico; detalla el plan de estudios de laboratorio por realizar inicialmente; plantea los distintos diagnósticos diferenciales; desarrolla aspectos relativos a evolución y pronóstico, y enumera los tratamientos disponibles para las formas agudas y las crónicas, así como para el manejo de las emergencias y en algunas situaciones especiales. Palabras clave: trombocitopenia inmune, pediatría, púrpura trombocitopénica idiopática, tratamiento.
\end{abstract}

\section{ABSTRACT}

Management, outcome, diagnosis, prognosis and treatment of immune thrombocytopenia are controversial. Several guidelines stating different experts' opinions have been published; however, noworldwide consensus regarding the management of the disease has still been reached. This guideline defines diagnostic criteria, states initial laboratory tests, establishes differential diagnosis, develops topics concerning outcome and prognosis, and enumerates available treatments for acute and chronic disease, as well as for management of life-threatening bleeding. Key words: immune thrombocytopenia, pediatrics, idiopathic thrombocytopenic purpura, treatment.

http: / / dx.doi.org/10.5546/ aap.2019.S243

Correspondencia: Dr. Hugo Donato: hcdonato@gmail.com

Cómo citar: Donato H, Bacciedoni V, Rapetti MC, Elena G, et al. Trombocitopenia inmune. Guía de diagnóstico y tratamiento. Arch Argent Pediatr 2019; 117 Supl 6: S243-S254.

\section{Financiamiento:}

Ninguno.

Conflicto de intereses:

Ninguno que declarar.

Recibido: 20-5-2019

Aceptado: 5-6-2019

\section{INTRODUCCIÓN}

La trombocitopenia inmune, denominación actual de la púrpura trombocitopénica inmune (PTI) según la nomenclatura más reciente, ${ }^{1}$ es una patología caracterizada por trombocitopenia aislada de origen autoinmune. En esta guía, se sigue utilizando la denominación de PTI debido a que está muy arraigada en los usos y prácticas de nuestro medio.

Tradicionalmente, se la consideró una enfermedad debida a la destrucción de plaquetas normales mediada por autoanticuerpos. Los avances que se han realizado en los últimos a ños en la investigación de los mecanismos fisiopatogénicos involucrados han llevado a una comprensión mucho mejor de la enfermedad. ${ }^{2-10}$ En la actualidad, es válido considerar la PTI como un síndrome, en el cual hay distintos mecanismos causales en los diversos individuos, que determinan la heterogeneidad de sus manifestaciones clínicas y las diferentes respuestas a los tratamientos disponibles, y que ocurre dentro de contextos genéticos determinados. Las alteraciones del sistema inmunológico son múltiples. Básicamente, un evento desencadenante primario (por mimetismo antigénico u otros mecanismos) origina una respuesta a toinmune que involucra mecanismos de inmunidad humoral y celular, y que es perpetuada por la deficiencia en los mecanismos regulatorios de células $T$, células $B$ 
y células dendríticas. La trombocitopenia es mediada por distintos mecanismos:

- La destrucción plaquetaria es causada, básicamente, por anticuerpos antiglicoproteínas plaquetarias (principalmente, II b/III a), pero también por citotoxicidad por células T CD8+.

- Estos mecanismos actúan, además, sobre los megacariocitos e inhiben la síntesis de plaquetas.

- Los niveles inadecuados de trombopoyetina contribuyen a esta menor síntesis.

- Hay evidencia que sugiere que algunos isotipos de anticuerpos no causan destrucción, sino que inhiben la activación plaquetaria.

Se han comunicado incidencias de 1,9 a 6,4 casos / 100000 personas / año en niños.

El manejo de la enfermedad es, actualmente, motivo de discusión en lo concerniente a la evolución, el diagnóstico, el pronóstico y el tratamiento. Se han publicado varias guías que expresan distintas opiniones de expertos, ${ }^{11-17}$ a pesar de lo cual no existe aún consenso mundial sobre el manejo más adecuado de esta patología.

Esta guía establece pautas para el manejo de la enfermedad en nuestro país. Para su confección, el panel se ha basado en los conceptos derivados de actualizaciones recientes sobre el tema y en la opinión de sus integrantes. Las recomendaciones están referidas, fundamentalmente, a PTI primaria, pero también a determinadas situaciones especiales y a algunas de las variantes secundarias de la enfermedad.

\section{DEFINICIÓN}

PTI primaria. Debe cumplir los cuatro requisitos siguientes: ${ }^{1,16,17}$

a. Trombocitopenia (recuento plaquetario menor de $\left.100 \times 10^{9} / 1\right)$.

b. Ausencia de enfermedad infecciosa aguda concomitante (p. ej., mononucleosis infecciosa, hepatitis).

c. Ausencia de patología sistémica de base (p. ej., lupus eritematoso sistémico, síndrome de inmunodeficiencia adquirida, linfoma).

d. Megacariocitos normales o aumentados en la médula ósea $\left({ }^{*}\right)$.

${ }^{*}$ ) Este criterio puede ser reemplazado por la ocurrencia de remisión completa espontánea o inducida por inmunoglobulina $G$ intravenosa (IGIV) en aquellos pacientes a los que no se les haya realizado la punción de la médula ósea.

PTI secundaria. Trombocitopenia (recuento plaquetario menor de $\left.100 \times 10^{9} / 1\right)$ asociada a alguna patología reconocible (como las indicadas en los puntos B y C). Para definirla, debe asociarse el nombre de la enfermedad de base al de PTI (p. ej., "PTI secundaria - asociada a lupus"). ${ }^{1}$

\section{PLAN DE ESTUDIOS AL MOMENTO DEL DIAGNÓSTICO}

Los estudios mínimos que se deben pedir al momento de la primera consulta son los siguientes: ${ }^{16,17}$

- Hemograma completo con recuento de plaquetas y visualización del frotis de sangre periférica.

- Coagulograma básico: tiempo de protrombina, tiempo parcial de tromboplastina activada, tiempo de trombina.

- Serología viral: las determinaciones mínimas por realizar serán para Epstein-Barr, virus de la inmunodeficiencia humana (VIH) y hepatitis. En forma opcional, es conveniente realizar otras determinaciones (citomegalovirus, virus del herpes, etc.).

- Prueba de Coombs directa.

- Medulograma (opcional). Puede obviarse si los restantes valores del hemograma son normales y mientras el paciente no reciba tratamiento con corticoesteroides. Si, a los 15 dias, el recuento plaquetario persiste en valores similares a los del momento del diagnóstico, deberá realizarse; si el recuento plaquetario experimentó un aumento parcial, quedará a criterio del médico tratante su realización posterior. También quedará a criterio del médico tratante su realización en cualquier momento en caso de que el paciente presente hemorragias graves o asociación con otra citopenia sin causa evidente.

- Estudio de colagenopatías: en pacientes $\geq 10$ años.

\section{DIAGNÓSTICO DIFERENCIAL}

Si la trombocitopenia se acompaña de anemia y/o leucopenia, deberán descartarse otras patologías causantes, fundamentalmente, leucemia aguda y aplasia medular. ${ }^{16,17}$ La punción y / o biopsia de la médula ósea, en estos casos, permitirá llegar al diagnóstico correcto.

Si el paciente presenta una trombocitopenia aislada, se deberá descartar que sea secundaria a una enfermedad de base. Las más frecuentes son lupus eritematoso, infección por VIH, mononucleosis infecciosa y linfoma no Hodgkin. Si los estudios complementarios correspondientes no arrojan resultados que muestren una causa evidente para la trombocitopenia, el diagnóstico diferencial se deberá realizar, fundamentalmente, 
con algunos síndromes de insuficiencia medular que pueden presentar trombocitopenia, como manifestación inicial o única, y con algunas trombocitopatías hereditarias que manifiestan trombocitopenia.

Dentro de los síndromes de insuficiencia medular, se deben considerar, sobre todo, la trombocitopenia amegacariocítica congénita y la trombocitopenia con ausencia de radio. En algunas oportunidades, las aplasias medulares, ya sean adquiridas o constitucionales (anemia de Fanconi), pueden comenzar con trombocitopenia aislada que se mantiene durante un tiempo más o menos prolongado hasta que se presentan las otras citopenias.

También, en estos casos, la punción y / o biopsia de la médula ósea permitirá llegar al diagnóstico correcto. El diagnóstico de las trombocitopatías hereditarias es complejo y debe recurrirse a estudios de funcionalidad plaquetaria (adhesividad y agregación), microscopía electrónica y detección de la mutación a nivel molecular.

\section{EVOLUCIÓN Y PRONÓSTICO}

Actualmente, se reconocen las siguientes formas de evolución: ${ }^{1}$

- PTI de reciente diagnóstico: Se alcanza la remisión completa (tres recuentos plaquetarios $\geq 100 \times 10^{9} / 1$ consecutivos) antes de los 3 meses de evolución. Se observa en, aproximadamente, el $50 \%$ de los casos.

- PTI persistente: Persisten recuentos plaquetarios $<100 \times 10^{9} / 1$ entre los 3 y los 12 meses de evolución. Se observa en alrededor del $30 \%$ de los casos.

- PTI crónica: Persisten recuentos plaquetarios $<100 \times 10^{9} / 1$ luego de los 12 meses de evolución. Se observa en, aproximadamente, el $20 \%$ de los casos. Un porcentaje importante de estos pacientes -el 20-50\% en distintas seriesalcanza la remisión completa espontánea luego de años de evolución. Se incluyen dentro de este grupo de pacientes a algunos casos (el 2-4 \% del total) en los cuales se produce una caída del recuento plaquetario por debajo de $100 \times 10^{9} / 1$ luego de haberse alcanzado la remisión completa y que esta se haya sostenido durante meses o años.

No existen, en la actualidad, indicadores clínicos o de laboratorio que, al momento del diagnóstico, permitan predecir con certeza cuál será la evolución. Sin embargo, hay varios factores que tienen distinto valor predictivo. ${ }^{18-33}$
El principal es la edad. Los niños menores de 12 meses constituyen un grupo pronóstico especial, caracterizado por la corta duración de la enfermedad y un muy elevado porcentaje de remisiones (aproximadamente, el $90 \%$ ), que no es influenciado por otros factores pronósticos. Por el contrario, en los pacientes $\geq 9$ años de edad, la enfermedad pasa a la cronicidad en más del $50 \%$ de los casos. Un recuento plaquetario inicial $<10 \times 10^{9} / 1$ y el antecedente de infección precedente en las 4 semanas previas son factores de pronóstico favorable en los niños mayores de 1 año de vida.

La muerte, debida a un sangrado grave en los órganos vitales -casi excluyentemente, hemorragia intracraneana-, se observa en alrededor del $0,2 \%$ de los pacientes. . $^{19,21,23-25,32,34,35}$

\section{TRATAMIENTO}

\section{Trombocitopenia inmune de reciente diagnóstico}

Recibirán tratamiento activo aquellos pacientes que presenten un recuento plaquetario $\leq 20 \times 10^{9} / 1$ y/o hemorragia activa y/o factores de riesgo (traumatismo craneoencefálico, politraumatismo, uso de antiagregantes en los 7-10 días previos, coagulopatía asociada, vasculitis). Se debe tener en cuenta que el único objetivo es inducir un aumento del recuento plaquetario que disminuya la gravedad del sangrado, ya que no existen, hasta ahora, medicamentos que actúen sobre el mecanismo primario de la enfermedad. La terapéutica será determinada por el médico tratante, dentro de las opciones que se muestran en la Tabla $1 .{ }^{11-13,15,36-44}$ En la Figura 1, se muestra el algoritmo recomendado para la secuencia terapéutica.

\section{Trombocitopenia inmune persistente}

Serán pasibles de tratamiento activo los pacientes cuyos recuentos plaquetarios se mantengan sostenidamente por debajo de $20 \times 10^{9} / 1$ o presenten hemorragias graves. Se podrá utilizar alguna de las opciones terapéuticas para PTI de reciente diagnóstico, solas o combinadas, con la finalidad de mantener recuentos plaquetarios por encima de los valores considerados seguros. En los casos graves o de sangrado intratable, se podrán considerar el uso de agonistas del receptor de trombopoyetina, la utilización de rituximab o la esplenectomía, según lo detallado para la PTI crónica. 
TABLA 1. Tratamiento de trombocitopenia inmune de reciente diagnóstico

\begin{tabular}{|c|c|c|c|c|}
\hline Tratamiento & Dosis & Tiempo de respuesta & Respuesta & Duración de la respuesta \\
\hline $\begin{array}{l}\text { Inmunoglobulina } \\
\text { G intravenosa }\end{array}$ & $\begin{array}{l}1 \text { g/kg/día } \\
\text { x } 2 \text { días. } \\
0,8 \text { g/ kg/día } \\
\text { x } 1 \text { día. }\end{array}$ & $\begin{array}{l}\text { 24-48 h. } \\
\text { Algo más tardía. }\end{array}$ & $90-95 \%$ & De 15 a 20 días. \\
\hline Prednisona & $\begin{array}{l}4 \mathrm{mg} / \mathrm{kg} \text { / día } \times 4 \text { días, } \\
\text { por vía oral } \\
\text { sis máxima de } 180 \mathrm{mg} / \text { día }) \text {. } \\
-2 \mathrm{mg} / \mathrm{kg} \text { / día } \times 14 \text { días, } \\
\text { por vía oral. }\end{array}$ & 3-5 días. & $80 \%$ & Generalmente, \\
\hline Metilprednisolona & $\begin{array}{l}30 \mathrm{mg} / \mathrm{kg} / \text { día } \\
3 \text { días, por vía intravenosa } \\
\text { (dosis máxima: } 1 \mathrm{~g} \text { ). }\end{array}$ & 3-5 días. & $60-90 \%$ & recaída rápida \\
\hline Dexametasona & $\begin{array}{l}\text { 20-40 mg/m²/ día, } \\
\text { por vía oral } \\
\text { is máxima: } 40 \mathrm{mg} \text { ) x } 4 \text { días. }\end{array}$ & 3-5 días. & $80 \%$ & \\
\hline $\begin{array}{l}\text { Inmunoglobulina } \\
\text { anti-D (en pacientes } \mathrm{Rh}+\text { ) }\end{array}$ & $\begin{array}{c}50-75 \mathrm{mcg} / \mathrm{kg} / \text { dosis, } \\
\text { por vía intravenosa. } \\
\text { Única dosis. }\end{array}$ & 4-5 días. & $80 \%$ & $\begin{array}{c}\text { De } 15 \text { a } 20 \text { días } \\
\text { Habitualmente, produce } \\
\text { un descenso de } \\
\text { hemoglobina de } 0,5-2 \mathrm{~g} / \mathrm{dl} \text {. }\end{array}$ \\
\hline Conducta expectante $\left(^{*}\right)$ & \multicolumn{3}{|c|}{$\begin{array}{l}\text { En pacientes sin sangrado o con sangrado mínimo } \\
\text { (solo manifestaciones en piel) y plaquetas }>20 \times 10^{9} / 1 \text {. }\end{array}$} & \\
\hline
\end{tabular}

FIGURA 1. Algoritmo para el tratamiento de trombocitopenia inmune de reciente diagnóstico

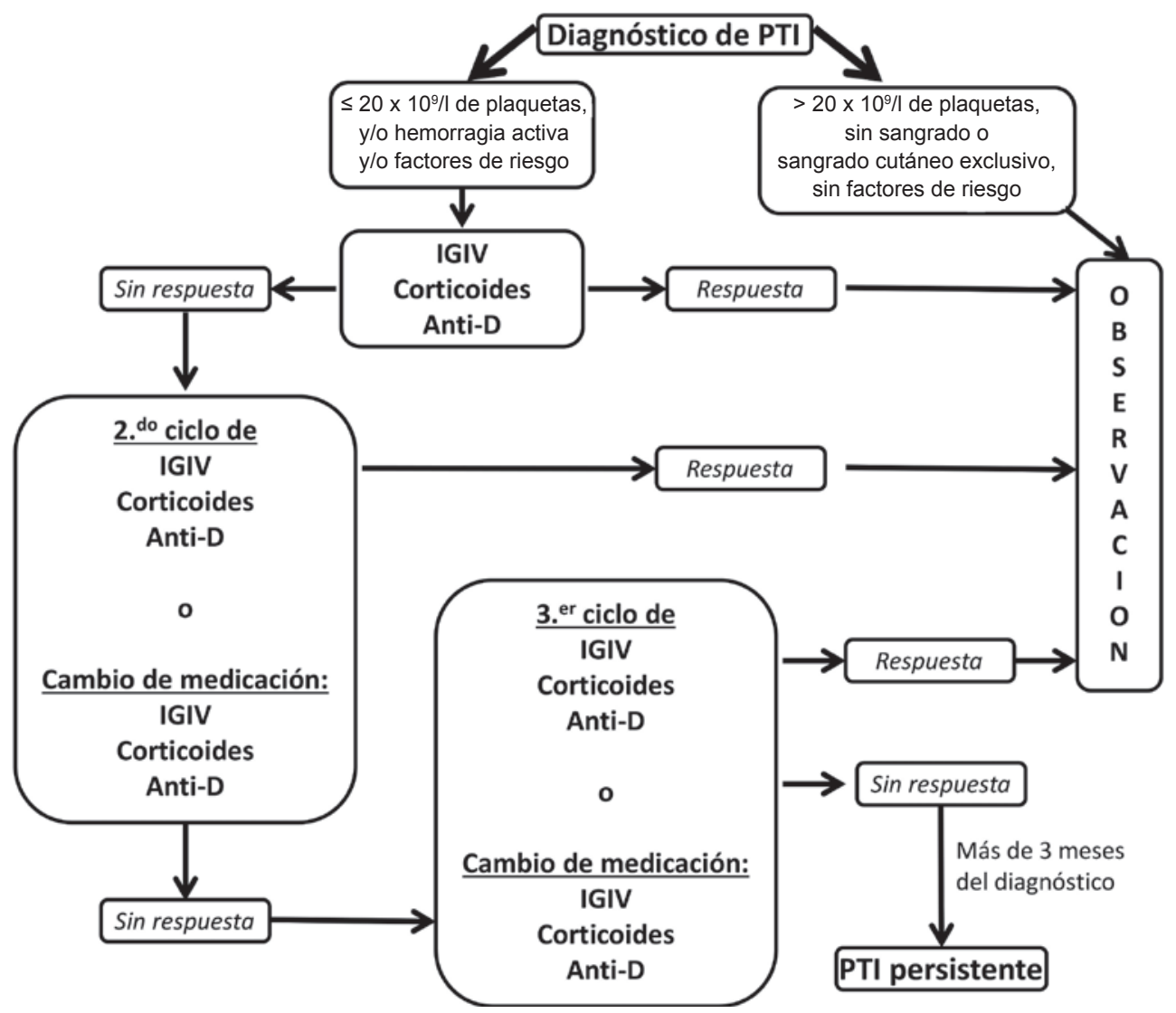

PTI: trombocitopenia inmune; IGIV: inmunoglobulina G intravenosa.

Fuente propia. 


\section{Trombocitopenia inmune crónica}

En los pacientes que mantengan recuentos plaquetarios por encima de $20 \times 10^{9} / 1$ y que no presenten sangrado activo, se recomienda la conducta expectante. Serán pasibles de tratamiento los pacientes cuyos recuentos plaquetarios se mantengan sostenidamente por debajo de $20 \times 10^{9} / 1$ o presenten hemorragias graves. Es recomendable que la conducta terapéutica por seguir sea consensuada entre el paciente, los padres y el médico tratante, luego de evaluar los distintos beneficios y riesgos de los tratamientos disponibles (Figura 2) ${ }^{45}$ Los tres factores más importantes que, por lo general, se toman en cuenta son los siguientes:

- Posibilidad de remisión espontanea tardía: Entre el $20 \%$ y el $30 \%$ de los pacientes con enfermedad crónica alcanzan la remisión sin tratamiento hasta varios años después del diagnóstico.

- Riesgo de muerte por la PTI: La incidencia de hemorragia intracraneana, causa casi excluyente de muerte en estos pacientes, oscila entre el $0,1 \%$ y el $0,5 \%$.

- Limitaciones en la calidad de vida, determinadas ya sea por la enfermedad o por las consecuencias de los tratamientos. ${ }^{46,47}$

Se podrá optar por administrar en forma periódica algunos de los tratamientos para PTI de reciente diagnóstico o por indicar otras terapias dentro de las siguientes opciones: $11-13,15,28,31,36-39,41,43,48-85$

\section{Esplenectomía:}

Actúa por un doble mecanismo. Fundamentalmente, por la eliminación del principal órgano donde se destruyen las plaquetas y, secundariamente, por la eliminación del principal órgano productor de anticuerpos. Produce la normalización del recuento plaquetario en el 70-80\% de los pacientes. La principal complicación de este tratamiento es el riesgo elevado de infección sistémica fulminante secundaria a la esplenectomía, que es mayor cuanto menor sea la edad del paciente. Por lo tanto, en los niños más pequeños, es conveniente postergarla hasta cumplir los 10 años; si no fuera factible, se tratará de realizarla después de cumplidos los 5 años; por debajo de esa edad, solo se esplenectomizarán aquellos pacientes que, a criterio del médico tratante, presenten factores de riesgo con potencial compromiso vital. En la Tabla 2, se muestran las ventajas y desventajas de la esplenectomía. ${ }^{76}$

Si las características del paciente lo permiten, la esplenectomía deberá realizarse, preferiblemente, por vía laparoscópica. ${ }^{77-80}$ Antes del procedimiento, el paciente deberá recibir, por lo menos, 2 semanas antes, vacunación contra Haemophilus influenzae, neumococo y meningococo. Luego de haberse realizado la esplenectomía, deberá recibir profilaxis diaria con penicilina o amoxicilina por vía oral, o mensual con penicilina benzatínica por vía intramuscular.

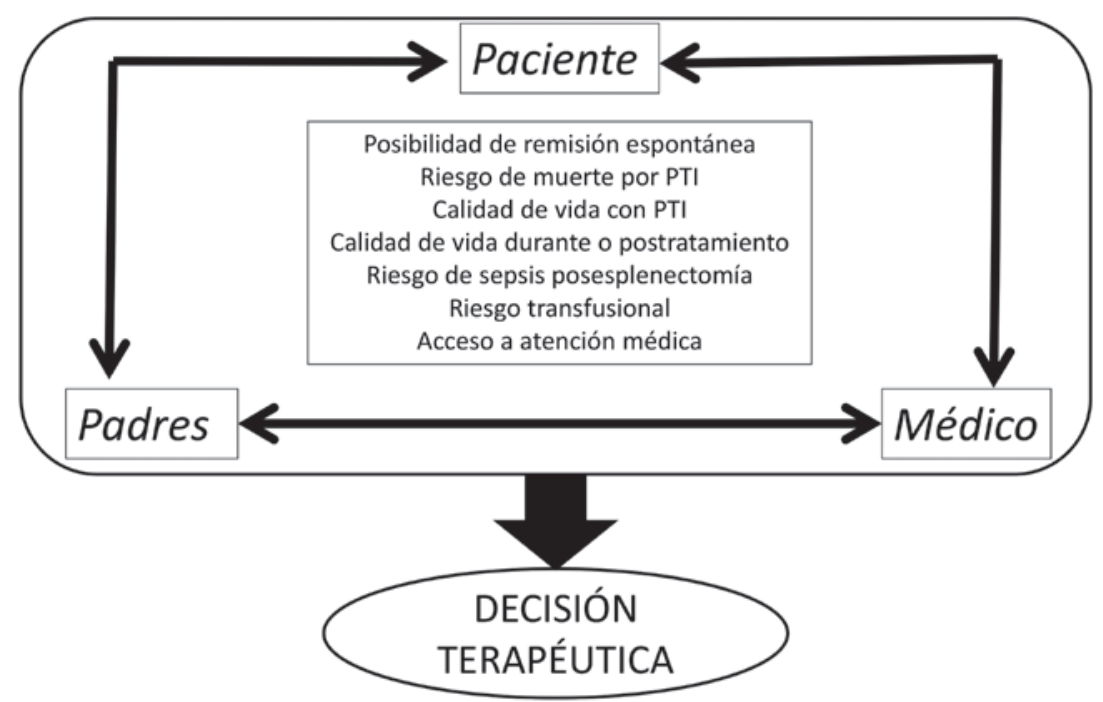

PTI: trombocitopenia inmune.

Fuente propia. 
Agonistas del receptor de trombopoyetina:

Actúan a nivel megacariocítico estimulando la síntesis de plaquetas. El objetivo del tratamiento con estas drogas es alcanzar y mantener en forma sostenida recuentos plaquetarios por encima de $50 \times 10^{9} / 1$. El porcentaje de respuesta inicial es de, aproximadamente, el $80 \%$ y el de respuesta sostenida a mediano/largo plazo, del 50-60 \% .

En la actualidad, existen dos opciones de tratamiento:

Eltrombopag: Está licenciado para uso pediátrico. Se administra por vía oral, diariamente, según las siguientes pautas:

- Pacientes de 1-5 años: $25 \mathrm{mg} /$ día.

- Pacientes $\geq 6$ años: $50 \mathrm{mg} /$ día.

- Pacientes del sudeste asiático: el $50 \%$ de la dosis.

- Pacientes > 6 años con compromiso hepático: el $50 \%$ de la dosis.

- Dosis máxima para administrar: $75 \mathrm{mg} /$ día.

- Los ajustes de dosis según el recuento plaquetario se realizan cada 2 semanas, con el aumento o la disminución de $25 \mathrm{mg} /$ día.

Romiplostim: No está licenciado para uso pediátrico; solo se puede indicar como tratamiento "compasivo". Se administra por vía subcutánea, una vez por semana. La dosis se debe ir ajustando semanalmente de acuerdo con el recuento plaquetario. En la Tabla 3, se muestran las pautas para el tratamiento con romiplostim.

En caso de no haber respuesta al tratamiento con uno de estos medicamentos, se deberá intentar con el otro.

\section{Rituximab:}

No está licenciado para uso pediátrico; solo se puede indicar como tratamiento "compasivo". Actúa, fundamentalmente, disminuyendo el título de anticuerpos por inhibición de síntesis de estos. Se utiliza a razón de $375 \mathrm{mg} / \mathrm{m}^{2} /$ dosis, una vez por semana, durante 4 semanas, aunque también se pueden indicar dosis menores. El porcentaje de respuestas favorables está en el orden del $35-40 \%$, pero el porcentaje de remisiones prolongadas (5 años) es solo del 20$25 \%$. Los efectos adversos graves, que obligan a suspender el tratamiento, se observan en, aproximadamente, el $4 \%$ de los pacientes.

Si la esplenectomía y/o los tratamientos con agonistas del receptor de trombopoyetina o con rituximab están contraindicados, fracasaron o no fueron aceptados, y el paciente mantiene recuentos plaquetarios $>20 \times 10^{9} / 1$ y no presenta hemorragias graves, se recomienda la conducta expectante y el uso sintomático de IGIV, corticoides o anti-D frente a episodios esporádicos de sangrado activo. Pero si el paciente persiste con recuentos plaquetarios $\leq 20 \times 10^{9} / 1$ o hemorragias graves, se podrá intentar con alguno de los tratamientos alternativos, según las pautas indicadas en la Tabla 4.

Se debe aclarar que, debido a la escasa experiencia pediátrica con estas terapias, los esquemas terapéuticos indicados son meramente tentativos. En la Figura 3, se muestra el algoritmo recomendado para la secuencia terapéutica en PTI crónica.

TABLA 2. Ventajas y desventajas de la esplenectomía

\begin{tabular}{|c|c|}
\hline A favor & En contra \\
\hline $\begin{array}{l}\text { "Cura" al 75-80\% de los pacientes. } \\
\text { Pocos efectos adversos. } \\
\text { Bajo riesgo de complicaciones posquirúrgicas tardías. } \\
\text { Baja incidencia de sepsis posesplenectomía }(0,1-0,5 \%) \text {. }\end{array}$ & $\begin{array}{c}\text { "Solo cura" al 75-80 \% de los pacientes. } \\
\text { Riesgo de hemorragia intraoperatoria. } \\
\text { Riesgo de tener que realizar esplenectomía accesoria. } \\
\text { Ausencia de un esquema óptimo determinado para la } \\
\text { profilaxis de la sepsis. } \\
\text { Riesgo aumentado de complicaciones trombóticas } \\
\text { a mediano/largo plazo. } \\
\text { Es una pérdida irreversible del órgano. }\end{array}$ \\
\hline
\end{tabular}

TABLA 3. Guía para el tratamiento con romiplostim

\begin{tabular}{lc}
\hline Recuento de plaquetas & Dosis \\
\hline$<50 \times 10^{9} / 1$. & $\begin{array}{l}\text { Inicial: } 1-3 \mathrm{mcg} / \mathrm{kg} / \mathrm{semana} . \\
<50 \times 10^{9} / 1 .\end{array}$ \\
$50-200 \times 10^{9} / 1$. & Se debe aumentar $1 \mathrm{mcg} / \mathrm{kg} / \mathrm{semana}$ \\
(dosis máxima: $10 \mathrm{mcg} / \mathrm{kg} / \mathrm{semana})$. \\
Se debe seguir con igual dosis. \\
$200-400 \times 10^{9} / 1$. & Se debe reducir de a $1 \mathrm{mcg} / \mathrm{kg} / \mathrm{semana}$. \\
$>400 \times 10^{9} / 1$. & Se debe suspender y reiniciar en una dosis menor si el \\
& recuento plaquetario desciende a $<200 \times 10^{9} / 1$.
\end{tabular}


TABLA 4. Opciones de tratamientos alternativos para trombocitopenia inmune refractaria

\begin{tabular}{|c|c|c|c|c|}
\hline Droga & Dosis & Respuesta & Tiempo hasta la respuesta & Observaciones \\
\hline Ciclofosfamida & $\begin{array}{l}1,5 \mathrm{~g} / \mathrm{m}^{2} / \text { dosis, } \\
\text { por vía intravenosa, } \\
\text { cada } 4 \text { semanas. }\end{array}$ & $50 \%$ & De 1 semana a 4 meses. & De 2 a 4 dosis en total. \\
\hline Azatioprina & 2-3 mg/kg/ día, por vía oral. & $60 \%$ & De 1 a 6 meses. & Dosis máxima: 150 mg/ día. \\
\hline $\begin{array}{l}\text { Micofenolato } \\
\text { Mofetil }\end{array}$ & $\begin{array}{l}1 \text { g, } 2 \text { veces por día, } \\
\text { por 3-4 semanas. }\end{array}$ & $45-75 \%$ & De 4 a 6 semanas. & \\
\hline Ciclosporina A & $\begin{array}{c}5 \mathrm{mg} / \mathrm{kg} / \text { día } \\
\text { la primera semana; } \\
\text { luego, } 2-3 \mathrm{mg} / \mathrm{kg} / \text { día, } \\
\text { por vía oral. }\end{array}$ & $50-80 \%$ & De 3 a 4 semanas. & $\begin{array}{c}\text { Se debe ajustar según } \\
\text { ciclosporinemia (hay que } \\
\text { mantener entre } 100 \text { y } \\
200 \mathrm{ng} / \mathrm{ml} \text { ). } \\
\text { Se debe vigilar la función } \\
\text { renal. }\end{array}$ \\
\hline Vincristina & $\begin{array}{c}0,02 \mathrm{mg} / \mathrm{kg} / \text { semana, } \\
\text { por vía intravenosa. }\end{array}$ & $10-75 \%$ & De 1 a 2 semanas. & $\begin{array}{c}\text { Dosis máxima: } \\
2 \text { mg. Total: } 3 \text { dosis. }\end{array}$ \\
\hline Vinblastina & $\begin{array}{l}0,1 \mathrm{mg} / \mathrm{kg} / \text { semana, } \\
\text { por vía intravenosa. }\end{array}$ & $10-75 \%$ & De 1 a 2 semanas. & $\begin{array}{l}\text { Dosis máxima: } 10 \mathrm{mg} \text {. } \\
\text { Total: } 3 \text { dosis. }\end{array}$ \\
\hline Danazol & $\begin{array}{l}2-3 \mathrm{mg} / \mathrm{kg} / \text { día, } \\
\text { por vía oral. }\end{array}$ & $40-60 \%$ & De 14 a 90 días. & \\
\hline
\end{tabular}

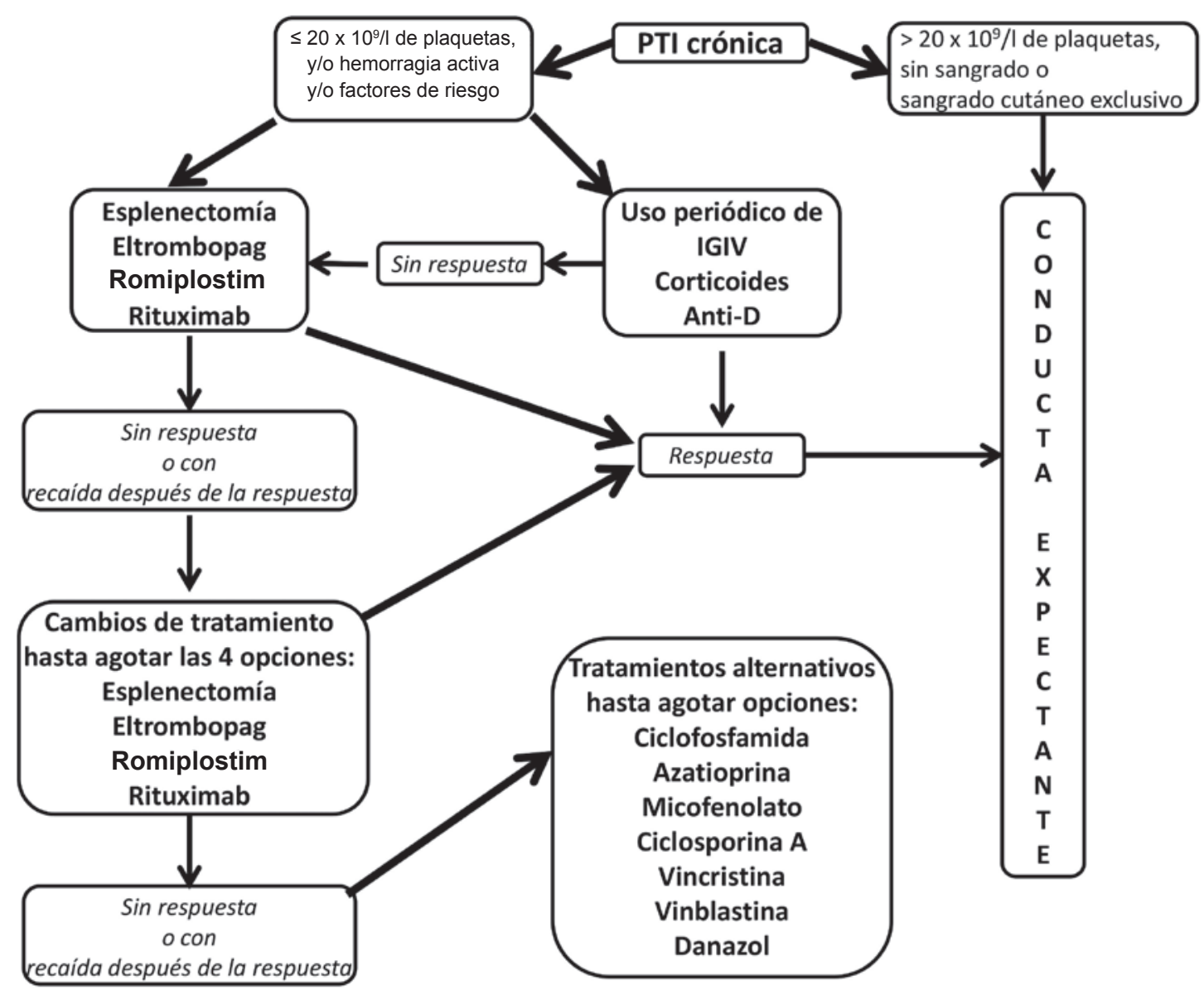

PTI: trombocitopenia inmune; IGIV: inmunoglobulina G intravenosa. 


\section{TRATAMIENTO DE EMERGENCIA}

Frente a una situación de hemorragia con riesgo de vida o de pérdida de un órgano o un miembro (hemorragia intracraneana, intraabdominal, ocular, síndrome compartimental $\mathrm{u}$ otras), que requiere el aumento inmediato del recuento de plaquetas, se deberán tomar las siguientes medidas en forma simultánea: ${ }^{11,17,36}$

- IGIV: $1 \mathrm{~g} / \mathrm{kg} /$ día por 1-2 días consecutivos.

- Metilprednisolona: $30 \mathrm{mg} / \mathrm{kg} / \mathrm{día}$, intravenosa, por 2-3 días consecutivos.

- Transfusión continua de concentrado de plaquetas: $0,5-1 \mathrm{U} / \mathrm{m}^{2} /$ hora o $2-4 \mathrm{U} / \mathrm{m}^{2}$ cada 6 horas.

- Control mecánico o quirúrgico en el sitio de sangrado, si es necesario y factible (abdomen, sistema nervioso central -SNC-).

- Esplenectomía de urgencia: será obligatoria en caso de sangrado que amerite una operación quirúrgica. Se realizará previamente a la cirugía en el sitio de sangrado (en caso de neurocirugía) o simultáneamente (en caso de laparotomía). En otros casos, se debe evaluar en forma individual la conveniencia de su realización ante el sangrado crítico persistente a pesar del tratamiento médico.

En casos refractarios al tratamiento anterior que tengan indicación de cirugía o presenten sangrado en el SNC no quirúrgico u otro sangrado crítico con imposibilidad de cirugía, se podrá utilizar factor VIIa recombinante. Dado que, para su eficacia, se requiere un número mínimo de plaquetas, se deberá administrar junto con concentrados plaquetarios.

\section{EFECTOS ADVERSOS DEL TRATAMIENTO}

Todos los medicamentos utilizados para el tratamiento de la PTI pueden producir efectos adversos graves. A continuación, se enumeran algunos de los más frecuentes o importantes causados por los medicamentos de uso habitual. ${ }^{17,36,66,68-70}$

- Corticoides: Osteoporosis, síndrome de Cushing, hipertensión arterial, hiperglucemia, cataratas, psicosis, otros.

- IGIV: Reacciones alérgicas, shock anafiláctico, meningitis aséptica, cefalea, transmisión de agentes infecciosos, otros.

- Inmunoglobulina anti-D: Reacciones alérgicas, hemólisis masiva, transmisión de agentes infecciosos, otros.

- Eltrombopag: Trastornos gastrointestinales, elevación de transaminasas, tromboembolismo, cataratas, mielofibrosis, nefrotoxicidad, otros.
- Romiplostim: Elevación de transaminasas, tromboembolismo, mielofibrosis, otros.

- Rituximab: Angioedema, enfermedad del suero, hipotensión arterial, broncoespasmo, edema laríngeo, neutropenia, otros.

- Drogas inmunosupresoras: Trastornos gastrointestinales, inmunosupresión, pancitopenia, alopecía, hepatotoxicidad, otros.

\section{TROMBOCITOPENIA INMUNE EN SITUACIONES ESPECIALES}

Trombocitopenia inmune y embarazo

La madre

La frecuencia de PTI gestacional oscila entre $1: 1000$ y $1: 10000$ embarazos. Representa el 3-5\% de las plaquetopenias durante el embarazo. El diagnóstico es por exclusión de otras causas más frecuentes de trombocitopenia gestacional (trombocitopenia incidental y desórdenes hipertensivos del embarazo). El embarazo, por su parte, puede agravar una PTI preexistente o reactivar una presuntamente curada. Para su tratamiento, se deben seguir las pautas habituales, pero enfatizando el manejo conservador, en especial, en el primero y segundo trimestre. ${ }^{17}$ El uso de corticoides durante el embarazo presenta complicaciones especiales que deben ser consideradas: diabetes gestacional, hipertensión, prematurez, abruptio placentae. Los agonistas del receptor de trombopoyetina están contraindicados en el primer trimestre, pero se ha descrito su uso más allá de la semana 20, al igual que para el rituximab. Micofenolato, danazol, ciclofosfamida y vincristina están contraindicados. La mortalidad materna de la PTI gestacional es casi nula. La morbilidad está dada por el recuento de plaquetas al momento del parto. Como regla general, el modo de parto (vaginal o cesárea) debe decidirse según el criterio obstétrico. Mientras que, para la madre trombocitopénica, la vía vaginal es, usualmente, más segura, no hay evidencia de que la cesárea sea más segura para el neonato trombocitopénico. En caso de parto vaginal, se debe minimizar el trauma fetal y evitar el uso de fórceps o vacuum.

\section{El recién nacido}

La trombocitopenia puede ocurrir tanto en hijos de madres con PTI activa diagnosticada antes del embarazo como en las diagnosticadas durante este, pero también en aquellas aparentemente curadas ya sea por remisión espontánea o por esplenectomía. ${ }^{86}$ La estimación más certera comunicada por los ensayos clínicos prospectivos de la incidencia de trombocitopenia 
grave (recuento plaquetario inferior a $50 \times 10^{9} / 1$ ) en los hijos de una madre con PTI es del $8 \%$ al $11 \%$. El nivel más bajo de plaquetas ocurre, habitualmente, 3-5 días después del nacimiento. No se ha encontrado correlación entre el recuento plaquetario del neonato y factores maternos, tales como recuento plaquetario, nivel de anticuerpos o antecedente de esplenectomía; solo la historia previa de trombocitopenia neonatal grave en un hermano es un factor predictivo importante. ${ }^{87}$ Las hemorragias, por lo general, son de leves a moderadas. La ocurrencia de hemorragias mayores (fundamentalmente, intracraneana) es inferior al $1 \%$. La trombocitopenia puede persistir hasta los 4 meses de vida, pero el riesgo de hemorragias disminuye casi totalmente después de las 2 primeras semanas de vida. No existe, hasta la fecha, ninguna guía basada en la evidencia para indicar el tratamiento en estos casos. En líneas generales, la conducta más conveniente para adoptar es la siguiente: ${ }^{17,86}$

Con recuento plaquetario $>50 \times 10^{9} / \mathrm{l}$ :

- Conducta expectante y control diario durante 7 días.

Con recuento plaquetario $<50 \times 10^{9} / \mathrm{l}$ :

- IGIV: $1 \mathrm{~g} / \mathrm{kg} /$ día x 1-2 días.

- Administración de plaquetas irradiadas si la gravedad del caso lo requiere.

- Ecografía transcraneal en todo neonato con plaquetopenia grave para excluir tempranamente el sangrado asintomático del SNC.

- Control periódico (frecuencia de acuerdo con la evolución) durante 4 semanas.

Se recomienda no aplicar vitamina $\mathrm{K}$ intramuscular hasta conocer el recuento plaquetario.

\section{Trombocitopenia inmune asociada a otras patologías (trombocitopenia inmune secundaria)}

Trombocitopenia inmune en hepatitis $C$

Durante el curso de la hepatitis por virus C, puede desarrollarse PTI secundaria. Los corticoides pueden provocar el aumento de la carga viral, mientras que los estudios con agonistas del receptor de trombopoyetina (solo en los adultos) demostraron eficacia, pero con riesgo de trombosis portal en enfermos con hipertensión portal y hepatopatía crónica no viral. Como pautas terapéuticas básicas, se recomiendan las siguientes: ${ }^{17}$

- Tratamiento antiviral si no hay contraindicación, con cuidadoso monitoreo del nivel de plaquetas.

- Si es necesario realizar tratamiento específico para la PTI, comenzar con IGIV.

- Realizar el seguimiento conjunto con Hepatología y/o Infectología.

\section{Trombocitopenia inmune en infección por virus de la inmunodeficiencia humana}

La infección por VIH puede acompañarse de PTI secundaria. El tratamiento antiviral puede mejorar el nivel de plaquetas. La utilización de corticoides, IGIV y anti-D ha demostrado seguridad y eficacia. La esplenectomía también es útil, pero sus riesgos no han sido bien establecidos. No se conoce con certeza el efecto que puedan tener los agonistas del receptor de trombopoyetina o el rituximab sobre la progresión de la infección viral. Como pautas terapéuticas básicas, se recomiendan las siguientes: ${ }^{17}$

- Tratamiento antiviral si no hay contraindicación.

- Si es necesario realizar tratamiento específico para la PTI, se pueden utilizar corticoides, IGIV, anti-D y esplenectomía.

- Realizar el seguimiento conjunto con Infectología.

\section{Trombocitopenia inmune en la infección por Helicobacter pylori}

La infección por $H$. pylori puede acompañarse de PTI secundaria. La terapia de erradicación de la bacteria puede mejorar el nivel de plaquetas en, aproximadamente, el $20 \%$ de los pacientes pediátricos afectados, en especial, en aquellos que tienen trombocitopenia leve y viven en áreas con alta prevalencia de la infección. ${ }^{88}$ Como pautas de manejo básicas, se recomiendan las siguientes: ${ }^{17}$

- Realizar la detección de la infección por $H$. pylori en los enfermos que más pueden beneficiarse de la terapia de erradicación $\mathrm{y} / \mathrm{o}$ en los no respondedores a las terapias farmacológicas de primera línea.

- El test de la urea en aire espirado, la detección del antígeno en materia fecal y la endoscopía son métodos más sensibles y específicos que la investigación de anticuerpos en sangre.

- En los enfermos en los que se demuestra la infección, comenzar con terapia de erradicación.

- Para el tratamiento de la trombocitopenia, se puede utilizar cualquiera de los tratamientos habituales. 


\section{Síndrome de Evans}

Es una rara enfermedad caracterizada por la aparición simultánea o secuencial de anemia hemolítica autoinmune, trombocitopenia inmune y / o neutropenia inmune. Constituye el 1-4 \% de los casos inicialmente diagnosticados como PTI o anemia hemolítica autoinmune. El 50-60 \% de los niños pueden superponer criterios de síndrome linfoproliferativo autoinmune (autoimmune lymphoproliferative syndrome; ALPS, por sus siglas en inglés). Otras afecciones asociadas al síndrome de Evans son inmunodeficiencia común variable, pos trasplante de órganos sólidos o médula ósea, exposición a fludarabina u otras drogas. La respuesta a los tratamientos habituales para PTI es menor $y$, frecuentemente, ineficaz. Tiene un alto porcentaje de mortalidad asociada a sangrado o sepsis.

\section{REFERENCIAS}

1. Rodeghiero F, Stasi R, Gernsheimer T, Michel M, et al. Standardization of terminology, definitions and outcome criteria in immune thrombocytopenic purpura of adults and children: report from an international working group. Blood. 2009; 113:(11)2386-93.

2. Cines DB, Bussel JB, Liebman HA, Luning Prak ET. The ITP syndrome: pathogenic and clinical diversity. Blood. 2009; 113(26):6511-21.

3. Stasi R. Immune thrombocytopenia: pathophysiologic and clinical update. Semin Thromb Hemost. 2012; 38(5):454-62.

4. Cines DB, Cuker A, Semple JW. Pathogenesis of immune thrombocytopenia. Presse Med. 2014; 43(4 Pt 2):e49-59.

5. Audia S, Mahévas M, Samson M, Godeau B, et al. Pathogenesis of immune thrombocytopenia. Autoimmun Rev. 2017; 16(6):620-32.

6. Emmons RV, Reid DM, Cohen RL, Meng G, et al. Human thrombopoietin levels are high when thrombocytopenia is due to megakaryocyte deficiency and low when due to increased platelet destruction. Blood. 1996; 87(10):4068-71.

7. Kosugi S, Kurata Y, Tomiyama Y, Tahara T, et al. Circulating thrombopoietin level in chronic immune thrombocytopenic purpura. Br J Haematol. 1996; 93(3):704-6.

8. Imbach P, Lazarus AH, Kühne T. Intravenous immunoglobulins induce potentially synergistic immunomodulations in autoimmune disorders. Vox Sang. 2010; 98(3 Pt 2):385-94.

9. Kappers-Klunne MC, De Haan M, Struijk PC, Van Vliet $\mathrm{HH}$. Serum thrombopoietin levels in relation to disease status in patients with immune thrombocytopenic purpura. Br J Haematol. 2001; 115(4):1004-6.

10. Hoemberg M, Stahl D, Schlenke P, Sibrowski W, et al. The isotype of autoantibodies influences the phagocytosis of antibody-coated platelets in autoimmune thrombocytopenic purpura. Scand J Immunol. 2011; 74(5):489-95.

11. George JN, Woolf SH, Raskob GE, Wasser JS, et al. Idiopathic thrombocytopenic purpura: a practice guideline developed by explicit methods for the American Society of Hematology. Blood. 1996; 88(1):3-40.

12. De Mattia D, Del Principe D, Del Vecchio GC, Jankovic $\mathrm{M}$, et al. Acute childhood idiopathic thrombocytopenic purpura: AIEOP consensus guidelines for diagnosis and treatment. Haematologica. 2000; 85(4):420-4.

13. British Committee for Standards in Haematology General Haematology Task Force. Guidelines for the investigation and management of idiopathic thrombocytopenic purpura in adults, children and in pregnancy. Br J Haematol. 2003; 120(4):574-96.

14. Shirahata A, Ishii E, Eguchi H, Okawa H, et al. Consensus guideline for diagnosis and treatment of childhood idiopathic thrombocytopenic purpura. Int J Hematol. 2006; 83(1):29-38.

15. Tarantino MD, Bolton-Maggs PH. Update on the management of immune thrombocytopenic purpura in children. Curr Opin Hematol. 2007; 14(5):526-34.

16. Comité Nacional de Hematología. Púrpura trombocitopénica inmunitaria. Guía de diagnóstico y tratamiento. Arch Argent Pediatr. 2010; 108(2):173-8.

17. Rapetti MC, Donato H, Fassi D, Fondevila C, et al. Trombocitopenia inmune. En:Sociedad Argentina de Hematología. Guías de Diagnóstico y Tratamiento. Buenos Aires: SAH; 2017.Págs.213-28.

18. Kühne T, Berchtold W, Tran VB, Tran VB, et al. Ethnicity and environment may affect the phenotype of immune thrombocytopenic purpura in children. Pediatr Res. 2000; 48(3):374-9.

19. Kühne T, Imbach P, Bolton-Maggs PHB, Berchtold W, et al. Newly diagnosed idiopathic thrombocytopenic purpura in childhood: an observational study. Lancet. 2001; 358(9299):2122-5.

20. Imbach P, Zimmerman S. Local and cultural aspects of childhood idiopathic thrombocytopenic purpura: a summary of statements from the 12 countries worldwide. J Pediatr Hematol Oncol. 2003; 25(Suppl 1):S68-73.

21. Kühne T, Buchanan GR, Zimmerman S, Michaels LA, et al. A prospective comparative study of 2540 infants and children with newly diagnosed idiopathic thrombocytopenic purpura (ITP) from the Intercontinental Childhood ITP Study Group. J Pediatr. 2003; 143(5):605-8.

22. DonatoH, Picón A, Rapetti MC, Rosso A, etal. Demographic data, outcome and prognosis of children with Idiopathic Thrombocytopenic Purpura(ITP) in Argentina. Results from a multicentered study. Hematol J. 2004; 5(Suppl 3):a482.

23. Watts RG. Idiopathic thrombocytopenic purpura: A 10-year natural history study at the Childrens Hospital of Alabama. Clin Pediatr (Phila). 2004; 43(8):691-702.

24. Imbach P, Kühne T, Muller D, Berchtold W, et al. Childhood ITP: 12 months follow-up data from the prospective Registry I of the Intercontinental Childhood ITP Study Group. Pediatr Blood Cancer. 2006; 46(3):351-6.

25. Donato H, Picón A, Martinez M, Rapetti MC, et al. Demographic data, natural history, and prognostic factors of idiopathic thrombocytopenic purpura in children: A multicentered study from Argentina. Pediatr Blood Cancer. 2009; 52(4):491-6.

26. Lowe EJ, Buchanan GR. Idiopathic thrombocytopenic purpura diagnosed during the second decade of life. J Pediatr. 2002; 141(2):253-8.

27. Sandoval C, Visintainer P, Ozkaynak MF, Tugal O, et al. Clinical features and treatment outcomes of 79 infants with immune thrombocytopenic purpura. Pediatr Blood Cancer. 2004; 42(1):109-12.

28. Donato H, Picón A, Rapetti MC, Rosso A, et al. Splenectomy and spontaneous remission in children with chronic idiopathic thrombocytopenic purpura. Pediatr Blood Cancer. 2006;47(Suppl 5):S737-9.

29. Glanz J, France E, Xu S, Hayes T, et al. A population-based, multisite cohort study of the predictors of chronic idiopathic thrombocytopenic purpura in children. Pediatrics. 2008; 121(3):e506-12.

30. Yacobovich J, Revel-Vilk S, Tamary H. Childhood immune thrombocytopenia - Who will spontaneously recover? Semin Hematol. 2013; 50(Suppl 1):S71-4. 
31. Edslev PW, Rosthøj S, Treutiger I, Rajantie J, et al. A clinical score predicting a brief and uneventful course of newly diagnosed idiopathic thrombocytopenic purpura in children. Br J Haematol. 2007; 138(4):513-6.

32. Schifferli A, Holbro A, Chitlur M, Coslovsky M, et al. A comparative prospective observational study of children and adults with immune thrombocytopenia: 2-year followup. Am J Hematol. 2018; 93(6):751-9.

33. Bennett CM, Neunert C, Grace RF, Buchanan G, et al. Predictors of remission in children with newly diagnosed immune thrombocytopenia: Data from the Intercontinental Cooperative ITP Study Group Registry II participants. Pediatr Blood Cancer. 2018; 65(1):e26736.

34. Butros LJ, Bussel JB. Intracranial hemorrhage in immune thrombocytopenic purpura: a retrospective analysis. $\mathrm{JPe}$ diatr Hematol Oncol. 2003; 25(8):660-4.

35. Psaila B, Petrovic A, Page LK, Menell J, et al. Intracranial hemorrhage (ICH) in children with immune thrombocytopenia (ITP): study of 40 cases. Blood. 2009; 114(23):4777-83.

36. Beardsley D, Nathan DG. Platelet abnormalities in infancy and childhood. En: Nathan DG, Orkin SH, Oski F (eds.). Nathan and Oski's Hematology of Infancy and Childhood. 5th ed. Philadelphia: WB Saunders; 1998.Págs.1585-1630.

37. Cines DB, Blanchette VS. Immune thrombocytopenic purpura. $N$ Engl J Med. 2002; 346(13):995-1008.

38. Imbach P, Barandun S, d'Apuzzo V, Baumgartner C, et al. High-dose intravenous gammaglobulin for idiopathic thrombocytopenic purpura in childhood. Lancet.1981; 1(8232):1228-31.

39. Salama A, Müeller-Eckhardt C, Kiefel V. Effect of intravenous immunoglobulin in immune thrombocytopenia. Lancet. 1983; 2(8343):193-5.

40. Scaradavou A, WooB, WoloskiBMR, Cunningham-Rundles $\mathrm{S}$, et al. Intravenous anti-D treatment of immune thrombocytopenic purpura: experience in 272 patients. Blood. 1997; 89(8):2689-700.

41. Semple JW, Allen D, Rutherford M, Woloski M, et al. Anti-D (WinRho SD) treatment of children with chronic autoimmune thrombocytopenic purpura stimulates transient cytokine/ chemokine production. Am J Hematol. 2002; 69(3):225-7.

42. Benesch M, KerblR, Lackner H, Berqhold A, et al. Low-dose versus high-dose immunoglobulin for primary treatment of acute immune thrombocytopenic purpura in children: results of a prospective, randomized single-center trial. J Pediatr Hematol Oncol. 2003; 25(10):797-800.

43. Beck CE, Nathan PC, Parkin PC, Blanchette VS, et al. Corticosteroids versus intravenous immune globulin for the treatment of acute immune thrombocytopenic purpura in children: A systematic review and meta-analysis of randomized controlled trials. J Pediatr. 2005; 147(4):521-7.

44. Lioger B, Maillot F, Ternant D, Passot C, et al. Efficacy and safety of anti-D immunoglobilins versus intravenous immunoglobulins for immune thrombocytopenia in children: Systematic review and meta-analysis of randomized controlled trials. J Pediatr. 2019; 204:225-33.e8.

45. Donato H. PTI. "Segun pasan los años". Conferencia Nacional Dr. Gregorio Bomchil. Hematología. 2017; 21(Extraordinario):137-51.

46. Flores A, Klaassen RJ, Buchanan G, Neunert CE. Patterns and influences in health-related quality of life in children with immune thrombocytopenia: A study from the Dallas ITP Cohort. Pediatr Blood Cancer. 2017; 64(8):e26405.

47. McMillan R, Bussel JB, George JN, Lalla D, et al. Self-reported health-related quality of life in adults with chronic immune thrombocytopenic purpura. Am J Hematol. 2008; 83(2):150-4.

48. Aronis S, Platokouki H, Mitsika A, Haidas S, et al. Seven- teen years of experience with chronic idiopathic thrombocytopenic purpura in childhood. Is therapy always better? Pediatr Hematol Oncol. 1994; 11(5):487-98.

49. Choudhry VP, Kashyap R, AhlawatS, Pati HP. Vinblastine and Danazol therapy in steroid resistant childhood chronic idiopathic thrombocytopenic purpura. Int J Hematol. 1995; 61(3):157-62.

50. Adams DM, Kinney TR, O'Branski-Rupp E, Ware RE. High-dose oral dexamethasone therapy for chronic childhood idiopathic thrombocytopenic purpura. J Pediatr.1996; 128(2):281-3.

51. Chen JS, Wu JM, Chen YJ, Yeh TF. Pulsed high-dose dexamethasone therapy in children with chronic idiopathic thrombocytopenic purpura. J Pediatr Hematol Oncol. 1997; 19(6):526-9.

52. Chandra J, Dua T, Narayan S, Jain V, et al. Dexamethasone therapy in chronic ITP. Indian Pediatr. 2000; 37(6):647-50.

53. Hemmila R, Foley DS, Castle VP, Hirschl RB. The response to splenectomy in pediatric patients with idiopathic thrombocytopenic purpura who fail high-dose intravenous immune globulin. J Pediatr Surg. 2000; 35(6):967-71.

54. Mantadakis E, Buchanan GR. Elective splenectomy in children with idiopathic thrombocytopenic purpura. J Pediatr Hematol Oncol. 2000; 22(2):148-53.

55. Tarantino MD. Treatment options for chronic immune (idiopathic) thrombocytopenia purpura in children. Semin Hematol. 2000; 3(1 Suppl 1):S35-41.

56. Gesundheit B, Cividalli G, Freeman A, Yatziv S, et al. Cyclosporin $\mathrm{A}$ in the treatment of refractory immune thrombocytopenia purpura in children. Eur J Haematol. 2001; 66(5):347-51.

57. El-Alfy MS, El-Tawil MM, Shahein N. 5- to 16- year followup following splenectomy in chronicimmune thrombocytopenic purpura in children. Acta Haematol. 2003; 110(1):20-4.

58. Wang J, Wiley JM, Luddy R, Greenberg J, et al. Chronic immune thrombocytopenic purpura in children: assessment of rituximab treatment. J Pediatr. 2005; 146(2):217-21.

59. Parodi E, Nobili B, Perrotta S, Rosaria Matarese SM, et al. Rituximab (Anti-CD20 monoclonal antibody) in children with chronic refractory symptomatic immune thrombocytopenic purpura: efficacy and safety of treatment. Int J Haematol. 2006; 84(1):48-53.

60. Peñalver FJ, Jimenez-Yuste $\mathrm{V}$, Almagro M, Alvarez-Larrán A, et al. Rituximab in the management of chronic immune thrombocytopenic purpura: an effective and safe therapeutic alternative in refractory patients. Ann Hematol. 2006; 85(6):400-6.

61. Tamminga RY, Bruin MC. Rituximab treatment for symptomatic chronic ITP. Pediatr Blood Cancer. 2006; 47(Suppl 5):S714-6.

62. Franchini M, Zaffanello M, Veneri D, Lippi G. Rituximab for the treatment of childhood chronic idiopathic thrombocytopenic purpura and hemophilia with inhibitors. Pediatr Blood Cancer. 2007; 49(1):6-10.

63. Kühne T, Blanchette V, Buchanan GR, Ramenghi U, et al. Splenectomy in children with idiopathic thrombocytopenic purpura: A prospective study of 134 children from the Intercontinental Childhood ITP Study Group. Pediatr Blood Cancer. 2007; 49(6):829-34.

64. Bennett CM, Rogers ZR, Kinnamon DD, Bussel JB, et al. Prospective phase $1 / 2$ study of rituximab in childhood and adolescent chronic immune thrombocytopenic purpura. Blood. 2006; 107(7):2639-42.

65. Chaturvedi S, Arnold DM, McCrae KR. Splenectomy for immune thrombocytopenia: down but not out. Blood. 2018; 131(11):1172-82.

66. Grainger JD, Locatelli F, Chotsampancharoen T, Donyush E, et al. Eltrombopag for children with chronic immune 
thrombocytopenia (PETIT2): a randomized, multicentre, placebo-controlled trial. Lancet. 2015; 386(10004):1649-58.

67. Elalfy MS, Abdelmaksoud AA, Eltonbary KY. Romiplostim in children with chronic refractory ITP: randomized placebo controlled study. Ann Hematol. 2011; 90(11):1341-4.

68. Bussel JB, Buchanan GR, Nugent DJ, Gnarra DJ, et al. A randomized, double-blind study of romiplostin to determine its safety and efficacy in children with immune thrombocytopenia. Blood. 2011; 118(1):28-36.

69. Bussel JB, Hsieh L, Buchanan GR, Stine K, et al. Long-term use of the thrombopoietin-mimetic romiplostim in children with severe chronic immune thrombocytopenia (ITP). Pediatr Blood Cancer. 2015; 62(2):208-13.

70. Cines DB, Wasser J, Rodeghiero F, Chong BH, et al. Safety and efficacy of romiplostim in splenectomized and nonsplenectomized patients with primary immune thrombocytopenia. Haematologica. 2017; 102(8):1342-51.

71. Khellaf M, Viallard JF, Hamidou M, Cheze S, et al. A retrospective pilot evaluation of switching thrombopoietic receptor-agonists in immune thrombocytopenia. Haematologica. 2013; 98(6):881-7.

72. Grace RF, Shimano KA, Bhat R, Neunert C, et al. Secondline treatments in children with immune thrombocytopenia: Effect on platelet count and patient-centered outcomes. Am J Hematol. 2019; 94(7):741-50.

73. Tumaini Massaro J, Chen Y, Ke Z. Efficacy and safety of thrombopoietin receptor agonists in children with chronic immune thrombocytopenia: meta-analysis. Platelets. 2019; Feb 27: 1-8. [Epub ahead of print].

74. Zhang J, Liang Y, Ai Y, Xie J, et al. Thrombopoietin-receptor agonists for children with immune thrombocytopenia: A systematic review. Expert Opin Pharmacother. 2017; 18(15):1543-51.

75. Grainger JD, Thind S. A practical guide to the use of eltrombopag in children with chronicimmune thrombocytopenia. Pediatr Hematol Oncol. 2017; 34(2):73-89.

76. Grainger JD, Bolton-Maggs PHB, Godeau B, Bussel J, et al. Diagnosis and management of chronic ITP: comments from an ICIS expert group. Ann Hematol. 2010; 89(Suppl 1):S11-7.

77. Danielson PD, ShaulDB, Phillipos JD, Stein JE, et al. Technical advances in pediatric laparoscopy have had a beneficial impact on splenectomy. J Pediatr Surg. 2000;35(11):1578-81.

78. Esposito C, Schaarschmidt K, Settimi A, Montupet P. Experience with laparoscopic splenectomy. J Pediatr Surg. 2001; 36(2):309-11.

79. Rescorla FJ, Engum SA, West KW, Tres Scherer LR 3rd, et al. Laparoscopic splenectomy has become the gold standard in children. Am Surg. 2002; 68(3):297-302.

80. Rosen M, Brody F, Walsh RM, Tarnoff M, et al. Outcome of laparoscopic splenectomy based on hematologic indication. Surg Endosc. 2002; 16(2):272-9.

81. Taube T, Schmid H, Reinhard H, Von Stackelberg A, et al. Effect of a simgle dose of rituximab in chronic immune thrombocytopenic purpura in childhood. Haematologica. 2005; 90(2):281-3.

82. Shenoy S, Kelly M, Grossman V, Lee S, et al. Rituximab therapy in children with chronic refractory immune cytopenia: Long-term efficacy and immune function analysis. Blood. 2003; 102:a1020.

83. Parodi E, Rivetti E, Amendola G, Bisogno G, et al. Longterm follow-up analysis after rituximab therapy in children with refractory symptomatic ITP: identification of factors predictive of a sustained response. Br J Haematol. 2008; 144(4):552-8.

84. Mueller BU, Bennett CM, Feldman HA, Bussel JB, et al. One year follow-up of children and adolescents with chronic immune thrombocytopenic purpura (ITP) treated with rituximab. Pediatr Blood Cancer. 2009; 52(2):259-62.

85. Moskowitz IPG, Gaynon PS, Shahidi NT, Cripe TP. Lowdose Cyclosporin A therapy in children with refractory immune thrombocytopenic purpura. J Pediatr Hematol Oncol. 1999; 21(1):77-9.

86. Donato H. Trastornos de las plaquetas. En: Donato H, Rapetti MC (eds.). Hematología Neonatal. Buenos Aires: FUNDASAP; 2007.Págs.227-54

87. Valat AS, CaulierMT, Devos P, Rugeri L, etal. Relationships between severe neonatal thrombocytopenia and maternal characteristics in pregnancies associated with autoimmune thrombocytopenia. Br J Haematol. 1998; 103(2):397-401.

88. Kim BJ, Kim HS, Jang HJ, Kim JH. Helicobacter pylori eradication in idiopathic thrombocytopenic purpura: A metaanalysis of randomized trials. Gastroenterol Res Pract. 2018; 2018:6090878. 